\title{
Factors influencing adherence to treatment in older adults with hypertension
}

This article was published in the following Dove Press journal:

Clinical Interventions in Aging

\author{
Bartosz Uchmanowicz' \\ Anna Chudiak' \\ Izabella Uchmanowicz' \\ Joanna Rosińczuk ${ }^{2}$ \\ Erika Sivarajan Froelicher ${ }^{3,4}$ \\ 'Division of Nursing in Internal \\ Medicine Procedures, Department \\ of Clinical Nursing, Faculty of Health \\ Sciences, Wroclaw Medical University, \\ Wroclaw, Poland; '2Department of \\ Nervous System Diseases, Faculty \\ of Health Sciences, Wroclaw \\ Medical University, Wroclaw, Poland; \\ ${ }^{3}$ Department of Physiological Nursing, \\ School of Nursing, University \\ of California San Francisco, San \\ Francisco, CA, USA; ${ }^{4}$ Department of \\ Epidemiology and Biostatistics, School \\ of Medicine, University of California \\ San Francisco, San Francisco, CA, USA
}

Correspondence: Izabella Uchmanowicz Division of Nursing in Internal Medicine Procedures, Department of Clinical Nursing, Faculty of Health Sciences, Wroclaw Medical University, 5 Bartla Street, 5I-618 Wroclaw, Poland Tel $+487 \mid 7841824$

Fax +48 7I 3459324

Email izabella.uchmanowicz@ umed.wroc.pl

\begin{abstract}
Purpose: Hypertension (HT) is considered to be the most common disorder in the general population. Demographic data indicate that older adults commonly suffer from HT. Older age is one of the key factors affecting the adherence of patients with HT. The main purpose was to identify demographic, socioeconomic, and clinical factors that affect adherence in older adults with HT. Materials and methods: This cross-sectional study included 150 patients ( 84 women and 66 men) with mean age of 72.1 years. The Hill-Bone Compliance to High Blood Pressure Therapy Scale (Hill-Bone CHBPTS) was used to evaluate the adherence to therapeutic recommendations for HT.
\end{abstract}

Results: The mean score obtained by the patients in the Hill-Bone CHBPTS was 20.19 (SD \pm 4.05$)$. The linear regression model showed the independent predictors of the total score $(P<0.05)$ : 1) age, each subsequent year of life raises the total score by an average of 0.2 points; 2 ) gender, males raise it by an average of 1.34 points compared to females; 3 ) education, a secondary, higher, or higher professional education lowers it by an average of 1.75 points compared to a primary education or no education; and 4) living with the family, having familial support lowers it by an average of 1.91 points compared to living alone or in an organized institution.

Conclusion: Our study has shown that the variables of age, education level, and living with the family were statistically significant in explaining the adherence rates. Health care professionals should pay more attention to older HT patients who have a low level of education and who experience the lack of social support. There is a need for a tailored education among this group of patients to better understand and adhere to medication treatment.

Keywords: elderly patients, hypertension, Hill-Bone scale, socioeconomic factors, clinical factors, cardiac nursing, health care providers

\section{Introduction}

Hypertension (HT) is considered to be the most common disorder in the general population and poses a health and economic problem worldwide. The definition given by the Writing Group of the American Society of Hypertension (ASH) states that HT is a progressive cardiovascular syndrome with many causes that result in both functional and structural changes to the heart and vascular systems. ${ }^{1}$

Therefore, attention should be paid to the overall assessment of the circulatory system, as well as other risk factors for the occurrence of this disease. In the worldwide population, HT affects $26.4 \%$ of people, and statistical projections indicate that by 2025, one-third of the population will suffer from HT. ${ }^{2}$ In the Polish population, HT occurs in $29 \%$ and is even more prevalent in the group of people older than 65 years, who account for over $50 \%$ of cases. ${ }^{3}$

Due to the changes associated with the aging process, older adults are especially prone to HT. Data from the Framingham Heart Study indicate that the onset of HT 
after 65 years of age affects $58.9 \%$ of the population and it affects over $70 \%$ of those aged 80 years or older. ${ }^{4}$

Treatment of older adults in accordance with the guidelines of the Polish Society of Hypertension (PSH), the European Society of Cardiology (ESC), and the European Society of Hypertension (ESH) greatly reduces the risk of strokes and the number of deaths. ${ }^{3}$ Regardless of age, blood pressure should be maintained below 130/80 mmHg. ${ }^{5}$

In older adults, it is recommended that antihypertensive therapy is conducted with caution and prudence due to atherosclerotic changes leading to myocardial ischemia and cerebral stroke. An overly rapid reduction in blood pressure can contribute to the diminished perfusion of vital organs and even aggravate ischemic changes. ${ }^{6}$

The condition for achieving the desired therapeutical effects is taking medications as prescribed and adhering to medical recommendations, as well as cooperating during every stage of the treatment. Unfortunately, the lack of cooperation and adherence to an accepted treatment regimen is one of the most challenging factors contributing to the low success of therapy in many chronic diseases. ${ }^{7}$

Many factors can affect the adherence in patients with HT. Among them are an older age, a low level of education, the possibility of side effects during pharmacotherapy, problems with memory or poor cognition, low socioeconomic status (unaffordability of medications), and the lack of proper instruction on the use of medicines from the health care professionals. ${ }^{8}$

It should be emphasized that the optimal control of blood pressure is extremely important for preventing complications. Unfortunately, because HT is asymptomatic, unless extremely high, patients often believe that there is no need for medication adherence, and that is why education is so very important, and this aspect needs to be included in the educational program and verified that the patient understands this point. Studies show that only a small proportion of patients with HT control their blood pressure, which results in low adherence to antihypertensive medications. ${ }^{9}, 10$ The factors influencing adherence can be divided into internal and external circumstances, as well as intentional and unintentional circumstances. ${ }^{11,12}$

AlGhurair et $\mathrm{al}^{13}$ demonstrated in their meta-analysis that adult adherence can be placed into these additional five categories: demographic, medical, medication, behavioral, and economic. The term "medication adherence" is defined as the extent to which a person's behavior agrees with the recommended medication regimen from the health care providers. ${ }^{14}$ The terms "compliance" and "adherence" are used interchangeably in medical nomenclature and refer to the patient-physician cooperation, adherence to medication, lifestyle modifications, and dietary plans. ${ }^{15}$

In the model by Leventhal and Cameron, ${ }^{16}$ medication adherence is a self-regulatory behavior. Moreover, patients often try to understand their illness by creating their own model by conceptualizing it as the representation of an illness. ${ }^{17}$ Unfortunately, this does not always lead to proper adherence. Adherence to therapeutic recommendations and cooperation with a therapeutic team reduce the risk of cardiovascular complications, decrease the expenditures associated with treatment, and improve the patients' quality of life (QOL). ${ }^{18}$

The patient-doctor cooperation affect was better adherence to recommendations, which has a positive affect on biopsychosocial functioning. Successful cooperation and perseverance have a positive effect on the course of the disease and add to the level of satisfaction for the patient and the entire therapeutic team. ${ }^{19}$

Considering the physiological changes in the human body and numerous comorbidities, the treatment of HT in the older adults is a great challenge for health care providers. Age may affect the pharmacokinetics of therapy and lessen the patient's ability to follow treatment recommendations. ${ }^{20}$

The level of adherence to recommendations and cooperation is a dynamic phenomenon during pharmacotherapy. It can change in a patient depending on the stage of treatment and in relation to the frequency of follow-up visits. It has been noted that patients are usually conscientious about taking their medication before the impending date of a visit to the doctor; after the visit, they return to their previous habits no matter what the health care providers' recommendations may be. Such variable behavior, in terms of adherence to therapeutic recommendations, is called "white coat adherence." ${ }^{21}$ The level of adherence also changes during the course of treatment to the disadvantage of patients in long-term treatment, and many reasons exist for diminished cooperation and adherence.

Risk factors for non-adherence to medical recommendations in antihypertensive therapy can be divided into four groups. The first factor related to the disease, such as the asymptomatic course of HT and the lack of immediate effects of the dose omission. Other factors are directly related to the patient, including failure to diagnose, social isolation, mental illness, a low level of awareness, a lack of knowledge about complications of untreated HT, a lack of active participation in therapy, and a failure to observe the dates of the follow-up visits. Declining levels of cooperation and adherence may 
also result due to the health care providers, not allowing enough time for the patient, the lack of therapy intensification, long waiting times for appointments, and the absence of proper communication between the health care providers and the patient. The last group of factors contributing to nonadherence is directly related to the therapy. The complexity of the dosing schedule discourages medications taking in accordance with the dosage directions, the duration of the treatment is long, there are negative effects for no treatment, side effects can occur, and there is a higher cost associated with treatment. ${ }^{22}$

There are many reasons for non-adherence to medical recommendations; however, some of them are directly related to the choice of appropriate antihypertensive therapy, including ineffectiveness, side effects, patient's low QOL, and the high cost of therapy. For this reason, a major role is that of the attending doctor who, when planning pharmacological treatment, should select drugs with the utmost care to eliminate risk factors that may contribute to therapeutic failure and encourage the patient to participate actively in the entire process. ${ }^{8,23}$

In this context, a true adherence concept is essential, which is defined as the extent to which the behavior corresponds with agreed recommendations from a health care provider. ${ }^{24}$ Non-adherence to medication may lead to increased morbidity, mortality, and additional costs to the health care system. Therefore, it is essential to know the patient's true adherence to medication, understand the causes of low adherence, and take actions to improve adherence. ${ }^{25}$ Therefore, the purpose of this study is to identify demographic, socioeconomic, and clinical factors that affect adherence in older adults with HT.

\section{Materials and methods Study design and patients}

This cross-sectional study included 150 patients hospitalized at the University Clinical Hospital in Wroclaw, Poland, due to the regular control of blood pressure visits. The mean age was 72.1 years $(\mathrm{SD} \pm 8.0)$, the mean duration of illness was 6 years $(\mathrm{SD} \pm 4.76)$, and the mean number of hospitalizations in previous years was $14.5(\mathrm{SD} \pm 9.39)$.

\section{Inclusion criteria}

Inclusion criteria to participate in the study were as follows: 1) an HT diagnosis based on the guidelines of the PSH, 2) HT lasting at least 12 months, 3) age $\geq 65$ years, 4) HT treated pharmacologically with at least one antihypertensive drug, 5) absence of cognitive impairment, and 6) mental disorders, as well as 7) the written informed consent of the patient to participate in this study.

\section{Ethical considerations}

The study protocol was approved by the Local Bioethics Committee at the Wroclaw Medical University, Poland (decision no KB-144/2016). Informed consent was obtained from all the patients. Each patient's right to withdraw from the study at any stage and the confidentiality of their responses were emphasized. The study was carried out in accordance with the tenets of the Declaration of Helsinki and Good Clinical Practice guidelines.

\section{Measurements}

Sociodemographic and clinical data were obtained from interviews performed by a specialist nurse and from patient records. The Hill-Bone Compliance to High Blood Pressure Therapy Scale (Hill-Bone CHBPTS) was used to measure adherence. ${ }^{26}$

The Hill-Bone CHBPTS questionnaire allows us to assess patients' adherence to recommendations for HT therapy. The scores of this questionnaire range from 14 to 56 points. The higher the number of points, the lower the level of adherence. It should be pointed out once again that giving the answer to the question "all the time" means that the patient is all the time non-compliant.

The Hill-Bone CHBPTS is a measure of antihypertensive treatment compliance that includes 14 items grouped into three subscales: 1) reduced sodium intake, 2) appointment keeping, and 3) medication taking. Each item is scored on a 4-point scale, response options consist of "none of the time $=1$ ", "some of the time $=2$ ", "most of the time $=3$ ", and "all the time $=4$ ". The global score may range between 14 and 56 points; the higher the score, the lower the treatment compliance. Giving the answer to the question, "all the time" means that the patient is all the time non-compliant. In this study, we used the Polish version of the Hill-Bone CHBPTS, which showed sufficient psychometric quality in different aspects of acceptability, reliability, and validity with the Cronbach's alpha score for the entire questionnaire at $0.851 .{ }^{27}$

\section{Statistical analyses}

Statistical analyses were performed with the use of R software version 3.5.0 (R Development Core Team, Foundation for Statistical Computing: Vienna, Austria). ${ }^{28}$ The normality of the distribution of variables was examined using the Shapiro-Wilk test. Medians and quartiles of distributions of 
specific variables were determined. A comparison between two groups was made using the Mann-Whitney test. A comparison between three and more groups was made using the Kruskal-Wallis test. When statistically significant differences were detected, post hoc analysis was performed with the Dunn test to identify statistically significant groups. Correlations between variables were obtained using Spearman's rank correlation coefficient. Multivariate analysis of the independent influences of many variables on the numerical variable was analyzed by linear regression. The results are presented as coefficients from a regression model with a $95 \%$ CI. The results are considered statistically significant with a $P$-value of $<0.05$.

\section{Results}

\section{Clinical and sociodemographic profile}

Detailed clinical and sociodemographic characteristics of the patients are summarized in Table 1.

Table I The clinical and sociodemographic characteristics of study patients

\begin{tabular}{|c|c|c|}
\hline Variables & Mean (SD) & Median (quartiles) \\
\hline Age (years) & $72.19(8)$ & $69(65-78)$ \\
\hline $\mathrm{SBP}(\mathrm{mmHg})$ & $14 \mid .84(\mid 7.21)$ & $140(130-150)$ \\
\hline $\mathrm{DBP}(\mathrm{mmHg})$ & 85.08 (12.99) & $90(80-90)$ \\
\hline BMI $\left(\mathrm{kg} / \mathrm{m}^{2}\right)$ & $27.96(5.63)$ & $27.38(24.8-30.79)$ \\
\hline Duration of disease (years) & $14.55(9.39)$ & $15(7-18)$ \\
\hline Features & $\mathbf{n}$ & $\%$ \\
\hline \multicolumn{3}{|l|}{ Sex } \\
\hline Female & 84 & 56.00 \\
\hline Male & 66 & 44.00 \\
\hline \multicolumn{3}{|l|}{ Marital status } \\
\hline Married or living together & 78 & 52.00 \\
\hline Single or divorced & 11 & 7.33 \\
\hline Widowed & 61 & 40.67 \\
\hline \multicolumn{3}{|l|}{ Education } \\
\hline Primary or none & 51 & 34.00 \\
\hline Secondary & 56 & 37.33 \\
\hline Vocational or higher & 43 & 28.67 \\
\hline \multicolumn{3}{|l|}{ Occupational activity } \\
\hline Employed & 34 & 22.67 \\
\hline Retired & 100 & 66.67 \\
\hline Annuitant & 16 & 10.67 \\
\hline \multicolumn{3}{|l|}{ Living arrangement } \\
\hline Living alone or in an organized institution & 52 & 34.67 \\
\hline Living with family & 98 & 65.33 \\
\hline \multicolumn{3}{|l|}{ JNC classification of $\mathrm{HT}$} \\
\hline Prehypertension & 38 & 25.33 \\
\hline Stage I HT & 81 & 54.00 \\
\hline Stage II HT & 31 & 20.67 \\
\hline \multicolumn{3}{|l|}{ Smoking } \\
\hline Non-smoker & 112 & 74.67 \\
\hline Ex-smoker & 25 & 16.67 \\
\hline Smoker & 13 & 8.67 \\
\hline \multicolumn{3}{|l|}{ Alcohol } \\
\hline Abstinent & 95 & 63.33 \\
\hline Non-abstinent & 55 & 36.67 \\
\hline
\end{tabular}


Table I (Continued)

\begin{tabular}{|c|c|c|}
\hline Variables & Mean (SD) & Median (quartiles) \\
\hline \multicolumn{3}{|l|}{ Comorbidities* } \\
\hline Diabetes & 80 & 53.33 \\
\hline Hypercholesterolemia & 48 & 32.00 \\
\hline Coronary artery disease & 46 & 30.67 \\
\hline Renal failure & 20 & 13.33 \\
\hline Rheumatic diseases & 25 & 16.67 \\
\hline \multicolumn{3}{|l|}{ Medications administration* } \\
\hline AA & 6 & 4.00 \\
\hline BA & 44 & 29.33 \\
\hline $\mathrm{CCB}$ & 42 & 28.00 \\
\hline ARB & 11 & 7.33 \\
\hline TD & 57 & 38.00 \\
\hline ACEI & 102 & 68.00 \\
\hline \multicolumn{3}{|l|}{ Therapy } \\
\hline Monotherapy & 67 & 44.67 \\
\hline Polytherapy & 83 & 55.33 \\
\hline
\end{tabular}

Note: *Multiple choice questions, percentages do not add up to $100 \%$.

Abbreviations: AA, alpha-adrenolytic; ACEI, angiotensin-converting enzyme inhibitor; ARB, angiotensin receptor blocker; BA, beta-adrenolytic; BMI, body mass index; CCB, calcium channel blocker; HT, hypertension; JNC, Joint National Committee; n, number of patients; TD, thiazide diuretic.

\section{Total score of the Hill-Bone CHBPTS}

The mean score obtained by the patients was $20.19(\mathrm{SD} \pm 4.05)$ and ranged from 14 to 32 points. The median was 19 points. The first and third quartiles were 17 and 22 , respectively, so the typical result in the analyzed group fell between 17 and 22 points (Table 2 ).

\section{Subscale results of the Hill-Bone CHBPTS}

Since each of the Hill-Bone CHBPTS subscales includes a different number of questions, each scale is composed of a different range of values. The reduced sodium intake subscale contains three questions (range, 3-12). Patients obtained on average 4.82 points $(\mathrm{SD} \pm 1.09)$ on this scale, which is an average of 1.61 points per question. The appointment keeping subscale includes two questions (range, 2-8), and patients obtained on average 3.33 points $(\mathrm{SD} \pm 1.19$ ), which is an average of 1.66 points per question. The medication taking subscale includes nine questions (range, 9-36), and patients obtained an average of 12.05 points ( $\mathrm{SD} \pm 3.08$ ), which is an average of 1.34 points per question.

Thus, the subjects demonstrated low compliance with appointment keeping and medication taking least (Table 3).

\section{Correlations of quantitative demographic and clinical factors with the Hill-Bone CHBPTS}

There were statistically significant $(P<0.05)$ correlation coefficients in the following comparisons: 1) the total Hill-Bone CHBPTS score is positively correlated with age and duration of disease and negatively with body mass index (BMI);2) the frequency of lower adherence regarding appointment keeping is positively correlated with age and duration of disease; and 3) the frequency of lower adherence regarding medication taking is positively correlated with age and duration of disease and negatively with BMI (Table 4).

Table 2 Total score of the Hill-Bone CHBPTS

\begin{tabular}{|c|c|c|c|c|c|c|}
\hline \multicolumn{7}{|c|}{ Total score of Hill-Bone CHBPTS $(n=\mid 50)$} \\
\hline Mean & SD & Median & Min & Max & Q I & Q3 \\
\hline 20.19 & 4.05 & 19 & 14 & 32 & 17 & 22 \\
\hline
\end{tabular}

Abbreviations: Hill-Bone CHBPTS, Hill-Bone Compliance to High Blood Pressure Therapy Scale; Max, maximum value; Min, minimum value; n, number of patients; QI, first quartile; Q3, third quartile. 
Table 3 Results for subscales of the Hill-Bone CHBPTS

\begin{tabular}{|l|l|l|l|l|l|l|l|}
\hline Hill-Bone CHBPTS subscale $\mathbf{( n = I 5 0 )}$ & Mean & SD & Median & Min & Max & QI & Q3 \\
\hline Reduced sodium intake & 4.82 & I.09 & 5 & 3 & 8 & 4 & 5 \\
\hline Appointment keeping & 3.33 & 1.19 & 3 & 2 & 6 & 2 & 4 \\
\hline Medication taking & 12.05 & 3.08 & II & 9 & 24 & 10 & 14 \\
\hline
\end{tabular}

Abbreviations: Hill-Bone CHBPTS, Hill-Bone Compliance to High Blood Pressure Therapy Scale; Max, maximum value; Min, minimum value; n, number of patients; Q I, first quartile; Q3, third quartile.

\section{Correlations of qualitative demographic and clinical factors with the Hill-Bone CHBPTS}

There were statistically significant $(P<0.05)$ differences in the following comparisons: 1) men more often disregarded recommendations about reduced sodium intake; 2) widowed people tended to be less adherent regarding medication taking and appointment keeping more often than people who were in relationships; 3) people with a higher education tended to be more adherent regarding medication taking, 4) people with a secondary education, basic education, or none education were more likely to have low adherence regarding appointment keeping than people with a higher education; 5) retirees were more likely to be low adherers on taking medications and on appointment keeping than occupationally active people; 6) people who living alone or in organized facilities have lower adherence level regarding appointment keeping and medication taking than people living within a family; 7) people with HT stage I more likely to have low adherence regarding medication taking than persons with HT stage II; 8) people with hypercholesterolemia were more likely to have low adherence regarding appointment keeping than people without hypercholesterolemia; 9) people with coronary artery disease (CAD) more likely to have low adherence regarding reduced sodium intake and appointment keeping than people without CAD; 10) people who take alphablockers had higher adherence to appointment keeping than the those who did not; 11) people who take thiazide diuretics were less adherent to taking medications than those who did not; and 12) people using polytherapy were less adherent to taking medications than those who used monotherapy (Table 5).

\section{The result of the multivariate analyses of sociodemographic and clinical factors on the Hill-Bone CHBPTS}

The linear regression model showed the independent predictors of the total score $(P<0.05)$ : 1$)$ age, each subsequent year of life raises the total score by an average of 0.2 points; 2) male gender, raise it by an average of 1.34 points as compared to females; 3 ) education, a secondary, higher, or higher professional education lowers it by an average of 1.75 points compared to a primary education or no education; and 4) living with the family, having familial support lowers the total score by an average of 1.91 points compared to living alone or in an organized institution (Table 6).

The $\mathrm{R}^{2}$ coefficient rise of $41.86 \%$ for this model means that $41.86 \%$ of the variation of the total score is explained by variables taken into the model. The remaining 58.14\% depends on variables not included in the model and on random factors.

\section{The influence of sociodemographic and clinical factors on the Hill-Bone CHBPTS - reduced sodium intake subscale}

The linear regression model showed the independent predictors of the total score $(P<0.05)$ : 1$)$ diastolic pressure, each additional millimeter of mercury lowers the total score by an average of 0.02 points; 2) retirement status lowers it by an average of 0.44 points compared to employed or retired patients; and 3) taking angiotensin-converting enzyme inhibitors, the medications raise it by an average of 0.65 points (Table 7 ).

Table 4 Correlations demographic and clinical factors (quantitative) with the Hill-Bone CHBPTS

\begin{tabular}{|c|c|c|c|c|c|}
\hline \multirow[t]{2}{*}{ Hill-Bone CHBPTS } & \multicolumn{5}{|c|}{ Correlation coefficient with** } \\
\hline & Age & SBP & DBP & BMI & $\begin{array}{l}\text { Duration of } \\
\text { disease }\end{array}$ \\
\hline Total score & $0.5 I^{*}$ & -0.03 & -0.13 & $-0.2 I^{*}$ & $0.30 *$ \\
\hline Reduced sodium intake & 0.04 & 0.06 & -0.09 & 0.01 & 0.01 \\
\hline Appointment keeping & $0.32 *$ & -0.05 & -0.08 & -0.07 & $0.25 *$ \\
\hline Medication taking & $0.50 *$ & -0.06 & -0.12 & $-0.27^{*}$ & $0.28^{*}$ \\
\hline
\end{tabular}

Notes: *Statistical significance for $P<0.05$. **Spearman's rank correlation coefficient (non-normal distributions of correlated variables)

Abbreviation: BMI, body mass index; Hill-Bone CHBPTS, Hill-Bone Compliance to High Blood Pressure Therapy Scale. 
Table 5 Results for demographic and clinical factors (qualitative)

\begin{tabular}{|c|c|c|c|c|}
\hline Group & Total score & Reduced sodium intake & Appointment keeping & Medication taking \\
\hline Female & $19(17-22)$ & $5(4-5)$ & $3(2-4)$ & $11(10-13.25)$ \\
\hline Male & $20(18-23)$ & $5(5-6)$ & $3.5(2-4)$ & II.5 (I0-14) \\
\hline$P$-value* & 0.22 & 0.01 & 0.71 & 0.36 \\
\hline Married or living together $(\mathrm{A})$ & $18(16.25-20)$ & $5(4-5)$ & $3(2-4)$ & $10(9-12)$ \\
\hline Single or divorced $(B)$ & $20(18-21.5)$ & $5(4.5-5)$ & $3(2.5-4)$ & $12(10.5-12.5)$ \\
\hline Widowed (C) & $22(19-23)$ & $5(4-5)$ & $4(3-4)$ & $13(10-15)$ \\
\hline$P$-value* & $<0.001$ & 0.752 & 0.04 & $<0.001$ \\
\hline Post hoc** & $C>A$ & - & $C>A$ & $C>A$ \\
\hline Primary or none $(\mathrm{A})$ & $22(18-24.5)$ & $5(4-6)$ & $3(2-4)$ & $13(10-15)$ \\
\hline Secondary (B) & $20(18-22)$ & $5(4-5)$ & $4(3-4)$ & $11(10-13)$ \\
\hline Vocational or higher $(C)$ & $18(16-19)$ & $5(4-5)$ & $2(2-4)$ & $10(9-11)$ \\
\hline$P$-value* & $<0.00$ & 0.38 & 0.01 & $<0.00$ \\
\hline Post hoc** & $A \cdot B>C$ & - & $A \cdot B>C$ & $A>B>C$ \\
\hline Employed (A) & $18(16-20)$ & $5(4-6)$ & $2.5(2-3.75)$ & $10(9-11.75)$ \\
\hline Retired (B) & $20(18-23)$ & $5(4-5)$ & $4(2-4)$ & $12(10-14)$ \\
\hline Annuitant (C) & $19(17.75-22)$ & $5(4.75-5)$ & $3(2-4)$ & $11.5(9.75-13)$ \\
\hline$P$-value* & 0.06 & 0.43 & 0.02 & 0.03 \\
\hline Post hoc** & - & - & $B>A$ & $B>A$ \\
\hline $\begin{array}{l}\text { Living alone or in an organized } \\
\text { institution (A) }\end{array}$ & $22(19-24)$ & $5(4-5)$ & $4(3-5)$ & $13(10-15)$ \\
\hline Living with family (B) & $18(|7-2|)$ & $5(4-5.75)$ & $3(2-4)$ & II (9-12.75) \\
\hline$P$-value* & $<0.00$ & 0.79 & $<0.00$ & $<0.00$ \\
\hline Prehypertension (A) & $19(16-22.75)$ & $4.5(4-5)$ & $3(2-4)$ & $11(10-13)$ \\
\hline Stage I HT (B) & $20(18-23)$ & $5(4-5)$ & $3(2-4)$ & $12(10-14)$ \\
\hline Stage II HT (C) & $18(17-20)$ & $5(4-5)$ & $3(2-4)$ & $10(9-12)$ \\
\hline$P$-value* & 0.043 & 0.164 & 0.808 & 0.025 \\
\hline Post hoc** & $B>C$ & - & - & $B>C$ \\
\hline Non-smoker & $20(18-23)$ & $5(4-5)$ & $3(2-4)$ & II (10-14) \\
\hline Ex-smoker & $18(|7-2|)$ & $5(4-5)$ & $3(2-4)$ & $10(9-13)$ \\
\hline Smoker & $18(17-20)$ & $5(4-6)$ & $3(2-4)$ & $10(9-12)$ \\
\hline$P$-value* & 0.066 & 0.127 & 0.456 & 0.052 \\
\hline Post hoc** & - & - & - & - \\
\hline Abstinent & $20(17-22)$ & $5(4-5)$ & $3(2-4)$ & $11(10-14)$ \\
\hline Non-abstinent & $19(17-22)$ & $5(4-6)$ & $3(2-4)$ & II (10-12.5) \\
\hline$P$-value* & 0.31 & 0.18 & 0.06 & 0.35 \\
\hline Lack of diabetes & $19(17-22)$ & $5(4-5)$ & $3(2-4)$ & II (9-14) \\
\hline Diabetes & $20(18-23)$ & $5(4-5.25)$ & $3(2-4)$ & $11(10-14)$ \\
\hline$P$-value* & 0.46 & 0.46 & 0.37 & 0.28 \\
\hline Lack of hypercholesterolemia & $19(17-23)$ & $5(4-5)$ & $3(2-4)$ & $11(10-14)$ \\
\hline Hypercholesterolemia & $20(18.75-22)$ & $5(4-5.25)$ & $4(3-5)$ & $11(10-13)$ \\
\hline$P$-value* & 0.09 & 0.5 & $<0.00$ & 0.95 \\
\hline Lack of coronary artery disease & $18(17-22)$ & $5(4-5)$ & $3(2-4)$ & II (9-13) \\
\hline Coronary artery disease & $21(19-23)$ & $5(4-5)$ & $4(3-5)$ & $13(10-14)$ \\
\hline$P$-value* & 0.00 & 0.78 & 0.01 & 0.01 \\
\hline Lack of renal failure & $19.5(17-22.75)$ & $5(4-5)$ & $3(2-4)$ & $11(10-14)$ \\
\hline Renal failure & $19(17.75-22)$ & $4.5(4-5.25)$ & $3(3-4)$ & $10.5(9-14)$ \\
\hline$P$-value* & 0.72 & 0.34 & 0.55 & 0.51 \\
\hline
\end{tabular}


Table 5 (Continued)

\begin{tabular}{|c|c|c|c|c|}
\hline Group & Total score & Reduced sodium intake & Appointment keeping & Medication taking \\
\hline Lack of rheumatic diseases & $19(17-22)$ & $5(4-5)$ & $3(2-4)$ & II (10-14) \\
\hline Rheumatic diseases & $21(18-22)$ & $5(4-5)$ & $3(3-5)$ & $12(10-13)$ \\
\hline$P$-value* & 0.32 & 0.66 & 0.33 & 0.62 \\
\hline Lack of AA & $20(17-22.25)$ & $5(4-5)$ & $3(2-4)$ & II (10-14) \\
\hline AA & $18(18-18.75)$ & $5(5-5)$ & $2(2-2.75)$ & $11(10.25-11.75)$ \\
\hline$P$-value* & 0.23 & 0.6 & 0.03 & 0.53 \\
\hline Lack of BA & $20(17-23)$ & $5(4-5)$ & $3(2-4)$ & II (10-14) \\
\hline BA & $19(17.75-22)$ & $5(4-6)$ & $3(2-4)$ & $11(10-13.25)$ \\
\hline$P$-value* & 0.47 & 0.87 & 0.08 & 0.66 \\
\hline Lack of CCB & $19.5(17-22)$ & $5(4-5)$ & $3(2-4)$ & $11(10-14)$ \\
\hline CCB & $19(17.25-22)$ & $5(4-5)$ & $3(2-4)$ & $12(10-13)$ \\
\hline$P$-value* & 0.95 & 0.69 & 0.49 & 0.99 \\
\hline Lack of ARB & $19(17-22)$ & $5(4-5)$ & $3(2-4)$ & $11(10-14)$ \\
\hline ARB & $23(17.5-25.5)$ & $5(4-5.5)$ & $4(3-4)$ & $14(10-16)$ \\
\hline$P$-value* & 0.17 & 0.81 & 0.4 & 0.14 \\
\hline Lack of TD & $19(17-21)$ & $5(4-5)$ & $3(2-4)$ & $10(9-13)$ \\
\hline TD & $22(18-24)$ & $5(4-5)$ & $4(2-4)$ & $13(11-15)$ \\
\hline$P$-value* & $<0.00$ & 0.32 & 0.17 & $<0.00$ \\
\hline Lack of ACEI & $20(18-23)$ & $5(4-6)$ & $3(2-4)$ & $12(10-14)$ \\
\hline ACEI & $19(17-22)$ & $5(4-5)$ & $3(2-4)$ & $11(9.25-13.75)$ \\
\hline$P$-value* & 0.15 & 0.25 & 0.71 & 0.13 \\
\hline Monotherapy & $19(|7-2|)$ & $5(4-5)$ & $3(2-4)$ & $10(10-13)$ \\
\hline Polytherapy & $20(18-23.5)$ & $5(4-5)$ & $3(2-4)$ & $12(10-14)$ \\
\hline$P$-value* & 0.08 & 0.54 & 0.40 & 0.04 \\
\hline
\end{tabular}

Notes: Data are represented as median (QI-Q3). *Mann-Whitney test and Kruskal-Wallis test. **The Dunn test (results for post hoc analysis). P, level of statistical significance. - , no relationship in post hoc analyses between $A, B$, and $C$ for reduced sodium intake subscale.

Abbreviations: AA, alpha-adrenolytic; $A C E I$, angiotensin-converting enzyme inhibitors; ARB, angiotensin receptor blocker; BA, beta-adrenolytic; $C C B$, calcium channel blocker; HT, hypertension; QI, first quartile; QI, third quartile; TD, thiazide diuretic.

Table 6 Results of multivariate analyses for general results of the Hill-Bone CHBPTS

\begin{tabular}{|c|c|c|c|c|}
\hline \multirow{2}{*}{$\begin{array}{l}\text { Variable } \\
\text { Age (years) }\end{array}$} & \multirow{2}{*}{$\begin{array}{l}\text { Coefficient } \\
0.20\end{array}$} & \multicolumn{2}{|c|}{$95 \% \mathrm{Cl}$} & \multirow{2}{*}{$\frac{P \text {-value }}{<0.01}$} \\
\hline & & 0.08 & 0.32 & \\
\hline $\mathrm{SBP}(\mathrm{mmHg})$ & -0.01 & -0.07 & 0.04 & 0.58 \\
\hline $\mathrm{DBP}(\mathrm{mmHg})$ & -0.02 & -0.08 & 0.04 & 0.56 \\
\hline BMI $\left(\mathrm{kg} / \mathrm{m}^{2}\right)$ & -0.03 & -0.15 & 0.09 & 0.62 \\
\hline Duration of disease (years) & 0.00 & -0.08 & 0.07 & 0.95 \\
\hline \multicolumn{5}{|l|}{ Sex } \\
\hline \multicolumn{5}{|l|}{ Female $=0$} \\
\hline Male $=I$ & 1.34 & 0.08 & 2.60 & 0.04 \\
\hline \multicolumn{5}{|l|}{ Marital status } \\
\hline \multicolumn{5}{|l|}{ Single, divorced, or widowed $=0$} \\
\hline Married or living together $=\mathrm{I}$ & 0.89 & -0.94 & 2.71 & 0.34 \\
\hline \multicolumn{5}{|l|}{ Education } \\
\hline \multicolumn{5}{|l|}{ Primary or none $=0$} \\
\hline Secondary, vocational, or higher=I & -1.75 & -3.22 & -0.28 & 0.02 \\
\hline
\end{tabular}


Table 6 (Continued)

\begin{tabular}{|c|c|c|c|c|}
\hline Variable & Coefficient & \multicolumn{2}{|c|}{$95 \% \mathrm{Cl}$} & $P$-value \\
\hline \multicolumn{5}{|l|}{ Occupational activity } \\
\hline \multicolumn{5}{|l|}{ Employed or annuitant $=0$} \\
\hline Retired $=$ I & -0.50 & -1.79 & 0.80 & 0.45 \\
\hline \multicolumn{5}{|l|}{ Living arrangement } \\
\hline \multicolumn{5}{|c|}{ Living alone or in an organized institution $=0$} \\
\hline Living with family $=I$ & -1.91 & -3.56 & -0.26 & 0.02 \\
\hline \multicolumn{5}{|l|}{ JNC classification of HT } \\
\hline \multicolumn{5}{|l|}{ Prehypertension $=0$} \\
\hline Stage I or II HT=I & 0.29 & -1.30 & 1.89 & 0.72 \\
\hline \multicolumn{5}{|l|}{ Smoking } \\
\hline \multicolumn{5}{|l|}{ Non-smoker $=0$} \\
\hline Smoker or ex-smoker=I & -1.08 & -2.49 & 0.32 & 0.13 \\
\hline \multicolumn{5}{|l|}{ Alcohol } \\
\hline \multicolumn{5}{|l|}{ Abstinent $=0$} \\
\hline Non-abstinent=I & 1.08 & -0.31 & 2.47 & 0.13 \\
\hline \multicolumn{5}{|l|}{ Diabetes } \\
\hline \multicolumn{5}{|l|}{$\mathrm{No}=0$} \\
\hline Yes $=I$ & 0.98 & -0.26 & 2.23 & 0.12 \\
\hline \multicolumn{5}{|l|}{ Hypercholesterolemia } \\
\hline \multicolumn{5}{|l|}{$\mathrm{No}=0$} \\
\hline Yes $=I$ & 0.56 & -0.77 & 1.89 & 0.41 \\
\hline \multicolumn{5}{|l|}{ Coronary artery disease } \\
\hline \multicolumn{5}{|l|}{$\mathrm{No}=0$} \\
\hline Yes $=I$ & 0.45 & -1.13 & 2.02 & 0.58 \\
\hline \multicolumn{5}{|l|}{ Renal failure } \\
\hline \multicolumn{5}{|l|}{$\mathrm{No}=0$} \\
\hline Yes $=1$ & -0.13 & -1.96 & I.7I & 0.89 \\
\hline \multicolumn{5}{|l|}{ Rheumatic diseases } \\
\hline \multicolumn{5}{|l|}{$\mathrm{No}=0$} \\
\hline Yes $=1$ & 0.95 & -0.68 & 2.58 & 0.25 \\
\hline \multicolumn{5}{|l|}{ Alpha-adrenolytic } \\
\hline \multicolumn{5}{|l|}{$\mathrm{No}=0$} \\
\hline Yes $=I$ & 0.12 & -1.42 & 1.65 & 0.88 \\
\hline \multicolumn{5}{|l|}{ BA } \\
\hline $\mathrm{No}=0$ & & & & \\
\hline Yes $=I$ & -0.91 & -4.16 & 2.33 & 0.58 \\
\hline $\mathrm{CCB}$ & & & & \\
\hline $\mathrm{No}=0$ & & & & \\
\hline Yes $=I$ & -0.01 & -1.63 & 1.62 & 0.99 \\
\hline ARB & & & & \\
\hline $\mathrm{No}=0$ & & & & \\
\hline Yes $=I$ & 0.84 & -1.00 & 2.69 & 0.37 \\
\hline TD & & & & \\
\hline $\mathrm{No}=0$ & & & & \\
\hline Yes $=1$ & 1.56 & $-|.4|$ & 4.52 & 0.30 \\
\hline
\end{tabular}

(Continued) 
Table 6 (Continued)

\begin{tabular}{|l|l|l|l|l|l|}
\hline Variable & Coefficient & $95 \%$ Cl & $P$-value \\
\hline ACEI & & & & \\
\hline No=0 & 1.20 & -0.58 & 2.98 & 0.19 \\
\hline Yes=I & \multicolumn{5}{|l|}{} \\
\hline Therapy & & & & \\
\hline Monotherapy=0 & 0.24 & -1.60 & 2.07 & 0.80 \\
\hline Polytherapy=I & \multicolumn{5}{|l|}{} \\
\hline
\end{tabular}

Note: $P$, level of statistical significance.

Abbreviations: AA, alpha-adrenolytic; ACEl, angiotensin-converting enzyme inhibitors; ARB, angiotensin receptor blocker; BA, beta-adrenolytic; BMI, body mass index; CCB, calcium channel blocker; Hill-Bone CHBPTS, Hill-Bone Compliance to High Blood Pressure Therapy Scale; HT, hypertension; JNC, Joint National Committee; TD, thiazide diuretic.

Table 7 Results of multivariate analyses for reduced sodium intake subscale of the Hill-Bone CHBPTS

\begin{tabular}{|c|c|c|c|c|}
\hline \multirow{2}{*}{$\begin{array}{l}\text { Variables } \\
\text { Age (years) }\end{array}$} & \multirow{2}{*}{$\begin{array}{l}\text { Coefficient } \\
-0.01\end{array}$} & \multicolumn{2}{|c|}{$95 \% \mathrm{Cl}$} & \multirow{2}{*}{$\begin{array}{l}P \text {-value } \\
0.78\end{array}$} \\
\hline & & -0.04 & 0.03 & \\
\hline $\mathrm{SBP}(\mathrm{mmHg})$ & 0.00 & -0.01 & 0.02 & 0.87 \\
\hline $\mathrm{DBP}(\mathrm{mmHg})$ & -0.02 & -0.04 & 0.00 & 0.02 \\
\hline BMI $\left(\mathrm{kg} / \mathrm{m}^{2}\right)$ & 0.02 & -0.02 & 0.05 & 0.35 \\
\hline Duration of disease (years) & 0.01 & -0.02 & 0.03 & 0.56 \\
\hline \multicolumn{5}{|l|}{ Sex } \\
\hline \multicolumn{5}{|l|}{ Female $=0$} \\
\hline Male $=I$ & 0.36 & -0.02 & 0.75 & 0.07 \\
\hline \multicolumn{5}{|l|}{ Marital status } \\
\hline \multicolumn{5}{|l|}{ Single, divorced, or widowed $=0$} \\
\hline Married or living together $=1$ & -0.46 & -1.02 & 0.10 & 0.11 \\
\hline \multicolumn{5}{|l|}{ Education } \\
\hline \multicolumn{5}{|l|}{ Primary or none $=I$} \\
\hline Secondary, vocational or higher=I & -0.31 & -0.76 & 0.14 & 0.18 \\
\hline \multicolumn{5}{|l|}{ Occupational activity } \\
\hline \multicolumn{5}{|l|}{ Employed or annuitant $=0$} \\
\hline Retired $=I$ & -0.44 & -0.84 & -0.04 & 0.03 \\
\hline \multicolumn{5}{|l|}{ Living arrangement } \\
\hline \multicolumn{5}{|c|}{ Living alone or in an organized institution $=0$} \\
\hline Living with family=I & 0.34 & -0.17 & 0.85 & 0.19 \\
\hline \multicolumn{5}{|l|}{ JNC classification of $\mathrm{HT}$} \\
\hline \multicolumn{5}{|l|}{ Prehypertension $=0$} \\
\hline Stage I or II HT=I & 0.44 & -0.05 & 0.93 & 0.08 \\
\hline \multicolumn{5}{|l|}{ Smoking } \\
\hline \multicolumn{5}{|l|}{ Non-smoker $=0$} \\
\hline Smoker or ex-smoker $=1$ & -0.18 & -0.61 & 0.25 & 0.42 \\
\hline \multicolumn{5}{|l|}{ Alcohol } \\
\hline \multicolumn{5}{|l|}{ Abstinent $=0$} \\
\hline Non-abstinent $=1$ & 0.29 & -0.14 & 0.71 & 0.19 \\
\hline \multicolumn{5}{|l|}{ Diabetes } \\
\hline \multicolumn{5}{|l|}{$\mathrm{No}=0$} \\
\hline Yes $=I$ & 0.34 & -0.04 & 0.72 & 0.08 \\
\hline \multicolumn{5}{|l|}{ Hypercholesterolemia } \\
\hline \multicolumn{5}{|l|}{$\mathrm{No}=0$} \\
\hline Yes $=I$ & 0.27 & -0.14 & 0.68 & 0.19 \\
\hline
\end{tabular}


Table 7 (Continued)

\begin{tabular}{|c|c|c|c|c|}
\hline Variables & Coefficient & \multicolumn{2}{|c|}{$95 \% \mathrm{Cl}$} & $P$-value \\
\hline \multicolumn{5}{|c|}{ Coronary artery disease } \\
\hline \multicolumn{5}{|l|}{$\mathrm{No}=0$} \\
\hline Yes $=I$ & 0.17 & -0.31 & 0.65 & 0.49 \\
\hline \multicolumn{5}{|l|}{ Renal failure } \\
\hline \multicolumn{5}{|l|}{$\mathrm{No}=0$} \\
\hline Yes $=I$ & -0.18 & -0.74 & 0.38 & 0.53 \\
\hline \multicolumn{5}{|l|}{ Rheumatic diseases } \\
\hline \multicolumn{5}{|l|}{$\mathrm{No}=0$} \\
\hline Yes $=I$ & 0.13 & -0.37 & 0.63 & 0.61 \\
\hline \multicolumn{5}{|l|}{ Alpha-adrenolytic } \\
\hline \multicolumn{5}{|l|}{$\mathrm{No}=0$} \\
\hline Yes $=I$ & -0.01 & -0.48 & 0.46 & 0.97 \\
\hline \multicolumn{5}{|l|}{$\mathrm{BA}$} \\
\hline \multicolumn{5}{|l|}{$\mathrm{No}=0$} \\
\hline Yes $=I$ & 0.26 & -0.74 & 1.25 & 0.61 \\
\hline \multicolumn{5}{|l|}{$\mathrm{CCB}$} \\
\hline \multicolumn{5}{|l|}{$\mathrm{No}=0$} \\
\hline Yes $=I$ & 0.27 & -0.22 & 0.77 & 0.28 \\
\hline \multicolumn{5}{|l|}{ ARB } \\
\hline \multicolumn{5}{|l|}{$\mathrm{No}=0$} \\
\hline Yes $=I$ & 0.52 & -0.04 & 1.09 & 0.07 \\
\hline \multicolumn{5}{|l|}{ TD } \\
\hline \multicolumn{5}{|l|}{$\mathrm{No}=0$} \\
\hline Yes $=I$ & 0.23 & -0.68 & 1.14 & 0.62 \\
\hline \multicolumn{5}{|l|}{ ACEI } \\
\hline \multicolumn{5}{|l|}{$\mathrm{No}=0$} \\
\hline Yes $=I$ & 0.65 & 0.11 & 1.20 & 0.02 \\
\hline \multicolumn{5}{|l|}{ Therapy } \\
\hline \multicolumn{5}{|l|}{ Monotherapy $=0$} \\
\hline Polytherapy $=$ I & 0.10 & -0.46 & 0.67 & 0.72 \\
\hline
\end{tabular}

Note: $P$, level of statistical significance.

Abbreviations: AA, alpha-adrenolytic; ACEl, angiotensin-converting-enzyme inhibitor; ARB, angiotensin receptor blocker; BA, beta-adrenolytic; BMI, body mass index; CCB, calcium channel blocker; Hill-Bone CHBPTS, Hill-Bone Compliance to High Blood Pressure Therapy Scale; HT, hypertension; JNC, Joint National Committee; TD, thiazide diuretic.

The $\mathrm{R}^{2}$ coefficient rise of $23.92 \%$ for this model means that $23.92 \%$ of the variation of the total score is explained by variables taken into the model. The remaining $76.08 \%$ depends on variables not included in the model and on random factors.

\section{The influence of sociodemographic and clinical factors on the Hill-Bone CHBPTS - appointment keeping subscale}

The linear regression model showed the independent predictors of the total score $(P<0.05)$ : 1$)$ age, each subsequent year of life raises the total score by an average of 0.04 points; 2) gender, males raise the total score by an average of
0.42 points compared to females; 3 ) marital status, being in relationship raises it by an average of 0.63 points compared to being single, divorced, or widowed; 4) living with family, having familial support lowers it by an average of 1.01 points compared to living alone or in an organized institution; 5) hypercholesterolemia, the condition raises it by an average of 0.81 points; 6 ) taking beta-adrenolytic, the medication lowers it by an average of 1.27 points; and 7) taking calcium channel blocker, the medication lowers it by an average of 0.64 points (Table 8 ).

The $\mathrm{R}^{2}$ coefficient rise of $36.37 \%$ for this model means that $36.37 \%$ of the variation of the total score is explained by variables taken into the model. The remaining $63.63 \%$ 
Table 8 Results of multivariate analyses for appointment keeping subscale of the Hill-Bone CHBPTS

\begin{tabular}{|c|c|c|c|c|}
\hline \multirow{2}{*}{$\begin{array}{l}\text { Variables } \\
\text { Age (years) }\end{array}$} & \multirow{2}{*}{$\begin{array}{l}\text { Coefficient } \\
0.04\end{array}$} & \multicolumn{2}{|c|}{$95 \% \mathrm{Cl}$} & \multirow{2}{*}{$\begin{array}{l}\boldsymbol{P} \text {-value } \\
0.03\end{array}$} \\
\hline & & 0.01 & 0.08 & \\
\hline $\mathrm{SBP}(\mathrm{mmHg})$ & 0.00 & -0.02 & 0.02 & 0.93 \\
\hline $\mathrm{DBP}(\mathrm{mmHg})$ & 0.00 & -0.02 & 0.02 & 1.00 \\
\hline BMI $\left(\mathrm{kg} / \mathrm{m}^{2}\right)$ & 0.00 & -0.04 & 0.04 & 0.96 \\
\hline Duration of disease (years) & -0.01 & -0.03 & 0.02 & 0.56 \\
\hline \multicolumn{5}{|l|}{ Sex } \\
\hline \multicolumn{5}{|l|}{ Female $=0$} \\
\hline Male $=1$ & 0.42 & 0.03 & 0.81 & 0.04 \\
\hline \multicolumn{5}{|l|}{ Marital status } \\
\hline \multicolumn{5}{|l|}{ Single, divorced, or widowed $=0$} \\
\hline Married or living together $=1$ & 0.63 & 0.07 & 1.19 & 0.03 \\
\hline \multicolumn{5}{|l|}{ Education } \\
\hline \multicolumn{5}{|l|}{ Primary or none $=I$} \\
\hline Secondary, vocational or higher=I & 0.25 & -0.20 & 0.70 & 0.27 \\
\hline \multicolumn{5}{|l|}{ Occupational activity } \\
\hline \multicolumn{5}{|l|}{ Employed or annuitant $=0$} \\
\hline Retired $=I$ & 0.13 & -0.26 & 0.53 & 0.51 \\
\hline \multicolumn{5}{|l|}{ Living arrangement } \\
\hline \multicolumn{5}{|c|}{ Living alone or in an organized institution $=0$} \\
\hline Living with family=I & -1.01 & $-1.5 \mid$ & -0.50 & $<0.01$ \\
\hline \multicolumn{5}{|l|}{ JNC classification of HT } \\
\hline \multicolumn{5}{|l|}{ Prehypertension=0 } \\
\hline Stage I or II HT=I & -0.08 & -0.57 & $0.4 \mathrm{I}$ & 0.74 \\
\hline \multicolumn{5}{|l|}{ Smoking } \\
\hline \multicolumn{5}{|l|}{ Non-smoker $=0$} \\
\hline Smoker or ex-smoker=1 & -0.17 & -0.60 & 0.27 & 0.45 \\
\hline \multicolumn{5}{|l|}{ Alcohol } \\
\hline \multicolumn{5}{|l|}{ Abstinent $=0$} \\
\hline Non-abstinent=I & -0.30 & -0.72 & 0.13 & 0.17 \\
\hline \multicolumn{5}{|l|}{ Diabetes } \\
\hline \multicolumn{5}{|l|}{$\mathrm{No}=0$} \\
\hline Yes $=I$ & 0.26 & -0.12 & 0.64 & 0.18 \\
\hline \multicolumn{5}{|l|}{ Hypercholesterolemia } \\
\hline \multicolumn{5}{|l|}{$\mathrm{No}=0$} \\
\hline Yes $=I$ & 0.81 & 0.41 & 1.22 & $<0.01$ \\
\hline Coronary artery disease & & & & \\
\hline $\mathrm{No}=0$ & & & & \\
\hline Yes $=I$ & -0.10 & -0.59 & 0.38 & 0.67 \\
\hline Renal failure & & & & \\
\hline $\mathrm{No}=0$ & & & & \\
\hline Yes $=I$ & 0.27 & -0.29 & 0.84 & 0.34 \\
\hline Rheumatic diseases & & & & \\
\hline $\mathrm{No}=0$ & & & & \\
\hline Yes $=1$ & 0.27 & -0.23 & 0.77 & 0.29 \\
\hline
\end{tabular}


Table 8 (Continued)

\begin{tabular}{|c|c|c|c|c|}
\hline Variables & Coefficient & \multicolumn{2}{|l|}{$95 \% \mathrm{Cl}$} & $P$-value \\
\hline \multicolumn{5}{|l|}{ Alpha-adrenolytic } \\
\hline \multicolumn{5}{|l|}{$\mathrm{No}=0$} \\
\hline Yes $=I$ & -0.17 & -0.64 & 0.31 & 0.49 \\
\hline \multicolumn{5}{|l|}{ BA } \\
\hline \multicolumn{5}{|l|}{$\mathrm{No}=0$} \\
\hline Yes $=I$ & -1.27 & -2.27 & -0.28 & 0.01 \\
\hline \multicolumn{5}{|l|}{$\mathrm{CCB}$} \\
\hline \multicolumn{5}{|l|}{$\mathrm{No}=0$} \\
\hline Yes $=I$ & -0.64 & -1.13 & -0.14 & 0.01 \\
\hline \multicolumn{5}{|l|}{ ARB } \\
\hline \multicolumn{5}{|l|}{$\mathrm{No}=0$} \\
\hline Yes $=I$ & -0.45 & -1.01 & 0.12 & 0.12 \\
\hline \multicolumn{5}{|l|}{ TD } \\
\hline \multicolumn{5}{|l|}{$\mathrm{No}=0$} \\
\hline Yes $=1$ & -0.13 & -1.04 & 0.78 & 0.78 \\
\hline \multicolumn{5}{|l|}{ ACEI } \\
\hline \multicolumn{5}{|l|}{$\mathrm{No}=0$} \\
\hline Yes $=I$ & -0.30 & -0.85 & 0.25 & 0.28 \\
\hline \multicolumn{5}{|l|}{ Therapy } \\
\hline \multicolumn{5}{|l|}{ Monotherapy $=0$} \\
\hline Polytherapy $=1$ & -0.40 & -0.96 & 0.17 & 0.17 \\
\hline
\end{tabular}

Note: $P$, level of statistical significance.

Abbreviations: AA, alpha-adrenolytic; ACEI, angiotensin-converting-enzyme inhibitor; ARB, angiotensin receptor blocker; BA, beta-adrenolytic; BMI, body mass index; CCB, Calcium channel blocker; Hill-Bone CHBPTS, Hill-Bone Compliance to High Blood Pressure Therapy Scale; HT, hypertension; JNC, Joint National Committee; TD, thiazide diuretic.

depends on variables not included in the model and on random factors.

\section{The influence of sociodemographic and clinical factors on the Hill-Bone CHBPTS - medication taking subscale}

The linear regression model showed the independent predictors of the total score $(P<0.05)$ : 1) age, each subsequent year of life raises the total score by an average of 0.16 points; 2) education, a secondary, higher, or higher professional education lowers it by an average of 1.69 points compared to primary education or no education; and 3) alcohol consumption, the condition raises it by an average of 1.09 points (Table 9).

The $\mathrm{R}^{2}$ coefficient rise of $43.17 \%$ for this model means that $43.17 \%$ of the variation of the total score was explained by variables taken into the model. The remaining 56.83\% depends on variables not included in the model and on random factors.

\section{Discussion}

Since patient medication adherence is a major health concern, we must articulate the significance of the problem and suggest improvements. Lack of adherence continues to present a problem for clinicians and researchers who need better information to understand medication adherence among older patients with HT. The goal of this study is to better understand the factors that influence the adherence situation regarding important variables. The key to effective antihypertensive therapy is to adhere to therapeutic recommendations and ensure appropriate cooperation with the entire medical team. Regarding the adherence to treatment requirements, the mean score in our study was 20.1 points out of 56 . The range of scores was 14-32 Higher results indicated less compliance and adherence to therapy. The literature on this subject provides several sociodemographic and clinical factors that affect the patients' adherence.

Some authors, such as Miller, ${ }^{29}$ Hyman and Pavlik, ${ }^{30}$ and Coons, ${ }^{31}$ have found that sociodemographic factors may 
Table 9 Results of multivariate analyses for medication taking subscale of the Hill-Bone CHBPTS

\begin{tabular}{|c|c|c|c|c|}
\hline \multirow{2}{*}{$\begin{array}{l}\text { Variables } \\
\text { Age (years) }\end{array}$} & \multirow{2}{*}{$\begin{array}{l}\text { Coefficient } \\
0.16 \\
\end{array}$} & \multicolumn{2}{|c|}{$95 \% \mathrm{Cl}$} & \multirow{2}{*}{$\begin{array}{l}\boldsymbol{P} \text {-value } \\
<0.01\end{array}$} \\
\hline & & 0.07 & 0.25 & \\
\hline $\mathrm{SBP}(\mathrm{mmHg})$ & -0.02 & -0.05 & 0.02 & 0.45 \\
\hline $\mathrm{DBP}(\mathrm{mmHg})$ & 0.00 & -0.04 & 0.05 & 0.87 \\
\hline BMI $\left(\mathrm{kg} / \mathrm{m}^{2}\right)$ & -0.05 & -0.14 & 0.04 & 0.29 \\
\hline Duration of disease (years) & 0.00 & -0.06 & 0.06 & 0.93 \\
\hline \multicolumn{5}{|l|}{ Sex } \\
\hline \multicolumn{5}{|l|}{ Female $=0$} \\
\hline Male $=I$ & 0.56 & -0.39 & 1.50 & 0.25 \\
\hline \multicolumn{5}{|l|}{ Marital status } \\
\hline \multicolumn{5}{|l|}{ Single, divorced, or widowed $=0$} \\
\hline Married or living together $=I$ & 0.72 & -0.65 & 2.09 & 0.30 \\
\hline \multicolumn{5}{|l|}{ Education } \\
\hline \multicolumn{5}{|l|}{ Primary or none $=1$} \\
\hline Secondary, vocational, or higher $=$ I & -1.69 & -2.80 & -0.59 & $<0.01$ \\
\hline \multicolumn{5}{|l|}{ Occupational activity } \\
\hline \multicolumn{5}{|l|}{ Employed or annuitant $=0$} \\
\hline Retired $=I$ & -0.19 & -1.17 & 0.78 & 0.69 \\
\hline \multicolumn{5}{|l|}{ Living arrangement } \\
\hline \multicolumn{5}{|c|}{ Living alone or in an organized institution $=0$} \\
\hline Living with family=I & -1.24 & -2.49 & 0.00 & 0.05 \\
\hline \multicolumn{5}{|l|}{ JNC classification of HT } \\
\hline \multicolumn{5}{|l|}{ Prehypertension $=0$} \\
\hline Stage I or II HT=I & -0.07 & -1.26 & 1.13 & 0.92 \\
\hline \multicolumn{5}{|l|}{ Smoking } \\
\hline \multicolumn{5}{|l|}{ Non-smoker $=0$} \\
\hline Smoker or ex-smoker=I & -0.74 & -1.79 & 0.32 & 0.17 \\
\hline \multicolumn{5}{|l|}{ Alcohol } \\
\hline \multicolumn{5}{|l|}{ Abstinent $=0$} \\
\hline Non-abstinent=I & 1.09 & 0.05 & 2.13 & 0.04 \\
\hline \multicolumn{5}{|l|}{ Diabetes } \\
\hline \multicolumn{5}{|l|}{$\mathrm{No}=0$} \\
\hline Yes $=I$ & 0.39 & -0.55 & 1.32 & 0.42 \\
\hline \multicolumn{5}{|l|}{ Hypercholesterolemia } \\
\hline \multicolumn{5}{|l|}{$\mathrm{No}=0$} \\
\hline Yes $=I$ & -0.53 & -1.52 & 0.47 & 0.30 \\
\hline Coronary artery disease & & & & \\
\hline $\mathrm{No}=0$ & & & & \\
\hline Yes $=I$ & 0.38 & -0.80 & 1.56 & 0.53 \\
\hline Renal failure & & & & \\
\hline $\mathrm{No}=0$ & & & & \\
\hline Yes $=1$ & -0.22 & -1.60 & 1.16 & 0.75 \\
\hline Rheumatic diseases & & & & \\
\hline $\mathrm{No}=0$ & & & & \\
\hline Yes $=I$ & 0.56 & -0.67 & 1.78 & 0.37 \\
\hline
\end{tabular}


Table 9 (Continued)

\begin{tabular}{|c|c|c|c|c|}
\hline Variables & Coefficient & \multicolumn{2}{|l|}{$95 \% \mathrm{Cl}$} & $P$-value \\
\hline \multicolumn{5}{|l|}{ Alpha-adrenolytic } \\
\hline \multicolumn{5}{|l|}{$\mathrm{No}=0$} \\
\hline Yes $=I$ & 0.29 & -0.86 & 1.44 & 0.62 \\
\hline \multicolumn{5}{|l|}{ BA } \\
\hline \multicolumn{5}{|l|}{$\mathrm{No}=0$} \\
\hline Yes $=I$ & 0.10 & -2.33 & 2.54 & 0.93 \\
\hline \multicolumn{5}{|l|}{$\mathrm{CCB}$} \\
\hline \multicolumn{5}{|l|}{$\mathrm{No}=0$} \\
\hline Yes $=I$ & 0.35 & -0.87 & 1.57 & 0.57 \\
\hline \multicolumn{5}{|l|}{ ARB } \\
\hline \multicolumn{5}{|l|}{$\mathrm{No}=0$} \\
\hline Yes $=I$ & 0.76 & -0.62 & 2.15 & 0.28 \\
\hline \multicolumn{5}{|l|}{ TD } \\
\hline \multicolumn{5}{|l|}{$\mathrm{No}=0$} \\
\hline Yes $=I$ & 1.46 & -0.78 & 3.69 & 0.20 \\
\hline \multicolumn{5}{|l|}{ ACEI } \\
\hline \multicolumn{5}{|l|}{$\mathrm{No}=0$} \\
\hline Yes $=I$ & 0.84 & -0.50 & 2.18 & 0.21 \\
\hline \multicolumn{5}{|l|}{ Therapy } \\
\hline \multicolumn{5}{|l|}{ Monotherapy $=0$} \\
\hline Polytherapy=I & 0.53 & -0.85 & 1.91 & 0.45 \\
\hline
\end{tabular}

Note: $P$, level of statistical significance.

Abbreviations: AA, alpha-adrenolytic; ACEI, angiotensin-converting enzyme inhibitor; ARB, angiotensin receptor blocker; BA, beta-adrenolytic; BMI, body mass index; CCB, calcium channel blocker; Hill-Bone CHBPTS, Hill-Bone Compliance to High Blood Pressure Therapy Scale; HT, hypertension; JNC, Joint National Committee; TD, thiazide diuretic.

affect adherence. These factors include age, gender, marital status, and educational level. The results of our study have confirmed their findings that age, gender, and educational level significantly associated with medication adherence.

According to $\mathrm{WHO},{ }^{32}$ adherence is modified by factors related to socioeconomic status, health care systems, health conditions, therapy, and patient-related factors. Therefore, research on adherence research in older adults is particularly important because this group of patients is often excluded from clinical trials. Because the older adults are prone to multiple comorbidities and present a higher risk of polypharmacy, they may have a lower adherence to a medication regimen. ${ }^{33-35}$

In the current study, older age, living alone, and female gender are determinants of the lower adherence measured by the Hill-Bone CHBPTS. Several studies have shown that it is probable that men will show better adherence than women. ${ }^{36,37}$ However, other studies have offered results that demonstrate that women have better adherence to antihypertensive therapy. ${ }^{38,39}$

A higher level of adherence to treatment recommendations in our study was observed in people living with a spouse or a partner, in patients who have a higher education level, and in people who work. This may be due to the support that patients receive from a close relationship; they may pay more attention to their health because they are thinking of their spouse or partner. Some studies have reported that a lack of a caregiver or a caregiver's large workload may influence adherence to treatment. ${ }^{40,41}$ In addition, patients receiving monotherapy adhere to antihypertensive therapies better, and these results are consistent with the research of other authors. ${ }^{42,43}$ Bangalore et $\mathrm{a}^{14}$ demonstrated that non-adherence is related to the increase in the number of pills that patients take. In the study conducted by Ingersoll and Cohen, ${ }^{45}$ it was noted that older adults are more likely to adhere to medication with a simpler dosing schedule rather than a complex dosing schedule.

Our study found that patients taking alpha-blockers present higher adherence to appointment keeping than the those who do not, and on the other hand, it was noted that patients who take thiazide diuretics are less adherent taking medications, and this may be resulted from higher risk for side effects and adverse reactions such as dizziness and light 
headedness, blurred vision, loss of appetite, itching, stomach upset, headache, or weakness.

Wang et $\mathrm{al}^{46}$ confirm that the number of tablets used during HT treatment should be kept to a minimum, especially in older adults. This practice gives an opportunity for increased adherence to therapeutic recommendations and a higher level of cooperation, as well as reducing the risk of the side effects of antihypertensive drugs. Undoubtedly, taking a large number of tablets each day does not encourage the patient to use pharmacotherapy, and the available data indicate that, in people older than 65 years, the number of medications that the patient takes per day varies from two to six doses. ${ }^{46}$

\section{Study limitations}

This study contains a few limitations. A cross-sectional study design at best provides clues to associations but limits definitive statements about causation. The most significant limitation is that the study sample was small and recruited from a single center. Another limitation is that the level of adherence in the study was measured using a direct method based on the self-reported questionnaire with a cross-sectional design of the study. Finally, no correction for multiple statistical comparisons was made.

\section{Practical implications}

The subject undertaken in the present study on factors influencing adherence to older adults with HT can be a valuable cognitive and practical cue for professional health care providers for patients in this clinical group. Identifying factors and understanding how they affect adherence in the elderly may allow optimizing care for patients with HT. It is an undisputed fact that age-related disorders may contribute to worse adherence. Support from medical staff is one of the factors that ensures adequate adherence for older adults.

\section{Conclusion}

This study analyzed the demographic, socioeconomic, and clinical variables that affect medication non-adherence in patients with HT. Our study has shown that the variables of age and education level were statistically significant in explaining the adherence rates. Therefore, when educating people about medications, medical teams should pay more attention to patients older than 65 years with HT, who have a low level of education and who lack social support. There is a need for focused and personalized education among this group of patients, so that they better understand and adhere to the medication treatments, with the goal to reduce the risk of stroke and myocardial infarction and their sequelae.

\section{Data sharing statement}

All coauthors have agreed to the submission and publication of this manuscript. The authors are able to provide a signed consent if required by the Journal Editor. The authors confirm that all data underlying the findings described in this manuscript are fully available to all interested researchers on request.

\section{Acknowledgments}

There were no other contributors to the article than the authors as well as there was no writing assistance regarding our paper. The certificated English language services were provided by an academic highly qualified native English speaker to ensure a proper language quality including syntax, spelling, and grammar issues. This study was conducted under a research project funded by the Ministry of Science and Higher Education in Poland as a part of the statutory grant for maintaining the research potential (no ST.E020.17.050).

\section{Author contributions}

All authors contributed to data analysis, drafting or revising the article, gave final approval of the version to be published, and agree to be accountable for all aspects of the work.

\section{Disclosure}

The authors report no conflicts of interest in this work.

\section{References}

1. Knypl K. Definition and distribution of hypertension - attitude the Writing Group of the American Society of Hypertension 2005. Relation from the 20. Congress of the American Society of Hypertension, San Francisco, 14-18 May 2005. Guide GPs. 2005;8(5):101-105.

2. Mills KT, Bundy JD, Kelly TN, et al. Global disparities of hypertension prevalence and control: a systematic analysis of population-based studies from 90 countries. Circulation. 2016;134(6):441-450.

3. Zdrojewski T, Rutkowski M, Bandosz P, et al. Prevalence and control of cardiovascular risk factors in Poland. Assumptions and objectives of the NATPOL 2011 Survey. Kardiol Pol. 2013;71(4):381-392.

4. Lloyd-Jones DM, Evans JC, Levy D. Epidemiology of hypertension in the old old: data from the community in the 1990s. Am J Hypertens. 2004; 17(5):200A.

5. Lin JD, Chen YL, Wu CZ, et al. Identification of normal blood pressure in different age group. Medicine. 2016;95(14):e3188.

6. Mancia G, Fagard R, Narkiewicz K, et al. 2013 ESH/ESC Guidelines for the management of arterial hypertension: the Task Force for the management of arterial hypertension of the European Society of Hypertension (ESH) and of the European Society of Cardiology (ESC). J Hypertens. 2013;31(7):1281-1357.

7. Vrijens B, de Geest S, Hughes DA, et al. A new taxonomy for describing and defining adherence to medications. Br J Clin Pharmacol. 2012; 73(5):691-705.

8. Kubica A, Ratajska A, Sinkiewicz W. Reasons of poor doctor-patient cooperation in chronic therapy. Folia Cardiol. 2010;5(2):78-83.

9. Borzecki AM, Oliveria SA, Berlowitz DR. Barriers to hypertension control. Am Heart J. 2005;149(5):785-794. 
10. Hassan NB, Hasanah CI, Foong K, et al. Identification of psychosocial factors of noncompliance in hypertensive patients. J Hum Hypertens. 2006;20(1):23-29.

11. Haynes RB, Ackloo E, Sahota N, McDonald HP, Yao X. Interventions for enhancing medication adherence. Cochrane Database Syst Rev. 2008; (2):CD000011.

12. Marshall IJ, Wolfe CD, McKevitt C. Lay perspectives on hypertension and drug adherence: systematic review of qualitative research. BMJ. 2012;345:e3953.

13. AlGhurair SA, Hughes CA, Simpson SH, Guirguis LM. A systematic review of patient self-reported barriers of adherence to antihypertensive medications using the world health organization multidimensional adherence model. J Clin Hypertens. 2012;14(12):877-886.

14. Leporini C, De Sarro G, Russo E. Adherence to therapy and adverse drug reactions: is there a link? Expert Opin Drug Saf. 2014;13(Suppl 1): 41-55.

15. Haynes RB, McDonald HP, Garg AX. Helping patients follow prescribed treatment: clinical applications. JAMA. 2002;288(22):2880-2883.

16. Leventhal H, Cameron L. Behavioral theories and the problem of compliance. Patient Educ Couns. 1987;10(2):117-138.

17. Kulkarni AS, Balkrishnan R, Anderson RT, Edin HM, Kirsch J, Stacy MA. Medication adherence and associated outcomes in medicare health maintenance organization-enrolled older adults with Parkinson's disease. Mov Disord. 2008;23(3):359-365.

18. Rycombel A, Lomper K, Uchmanowicz I. Adherence and compliance in the treatment of hypertension. Arter Hypertens. 2014;18(3): $151-158$.

19. Guerrero D, Rudd P, Bryant-Kosling C, Middleton BF. Antihypertensive medication-taking investigation of a simple regimen. Am J Hypertens. 1993;6(7 Pt 1):586-592.

20. Leenen FH, Coletta E. Pharmacokinetic and antihypertensive profile of amlodipine and felodipine-ER in younger versus older patients with hypertension. J Cardiovasc Pharmacol. 2010;56(6):669-675.

21. Hughes DA, Bagust A, Haycox A, Walley T. The impact of noncompliance on the cost-effectiveness of pharmaceuticals: a review of the literature. Health Econ. 2001;10(7):601-615.

22. Ambrosioni E, Leonetti G, Pessina AC, Rappelli A, Trimarco B, Zanchetti A. Patterns of hypertension management in Italy: results of a pharmacoepidemiological survey on antihypertensive therapy. Scientific Committee of the Italian Pharmacoepidemiological Survey on Antihypertensive Therapy. J Hypertens. 2000;18(11):1691-1699.

23. Osterberg L, Blaschke T. Adherence to medication. N Engl J Med. 2005; 353(5):487-497.

24. Nieuwenhuis MM, Jaarsma T, van Veldhuisen DJ, van der Wal MH Self-reported versus "true" adherence in heart failure patients: a study using the Medication Event Monitoring System. Neth Heart J. 2012; 20(7-8):313-319.

25. Kubica A, Gruchała M, Jaguszewski M, et al. Adherence to treatmenta pivotal issue in long-term treatment of patients with cardiovascular diseases. An expert standpoint. Med Res J. 2017;2(4):123-127.

26. Kim MT, Hill MN, Bone LR, Levine DM. Development and testing of the hill-bone compliance to high blood pressure therapy scale. Prog Cardiovasc Nurs. 2000;15(3):90-96.

27. Uchmanowicz I, Jankowska-Polańska B, Chudiak A, SzymańskaChabowska A, Mazur G. Psychometric evaluation of the polish adaptation of the hill-bone compliance to high blood pressure therapy scale. BMC Cardiovasc Disord. 2016;16:87.

Clinical Interventions in Aging

\section{Publish your work in this journal}

Clinical Interventions in Aging is an international, peer-reviewed journa focusing on evidence-based reports on the value or lack thereof of treatments intended to prevent or delay the onset of maladaptive correlates of aging in human beings. This journal is indexed on PubMed Central, MedLine,
28. Lumley T, Sebestyen K, Lober WB, Painter I. An open source environment for the statistical evaluation of outbreak detection methods. AMIA Annu Symp Proc. 2005;2005:1037.

29. Miller TA. Health literacy and adherence to medical treatment in chronic and acute illness: a meta-analysis. Patient Educ Couns. 2016;99(7): 1079-1086.

30. Hyman DJ, Pavlik V. Medication adherence and resistant hypertension. J Hum Hypertens. 2015;29(4):213-218.

31. Coons SJ. Medication compliance: the search for answers continues. Clin Ther. 2001;23(8):1294-1295.

32. Sabaté E [webpage on the Internet]. Project WA to LTT, Network GAI, Diseases WHOD of M of N. Adherence to long-term therapies: evidence for action; 2003. Available from: http://apps.who.int/iris/ handle/10665/42682. Accessed July 2, 2018.

33. Toh MR, Teo V, Kwan YH, Raaj S, Tan SY, Tan JZ. Association between number of doses per day, number of medications and patient's non-compliance, and frequency of readmissions in a multi-ethnic Asian population. Prev Med Rep. 2014;1:43-47.

34. Toh SG, Ang E, Devi MK. Systematic review on the relationship between the nursing shortage and job satisfaction, stress and burnout levels among nurses in oncology/haematology settings. Int J Evid Based Healthc. 2012;10(2):126-141.

35. Yap AF, Thirumoorthy T, Kwan YH. Medication adherence in the elderly. Geriatr Gerontol Int. 2016;7(2):64-67.

36. Degli Esposti E, Sturani A, Di Martino M, et al. Long-term persistence with antihypertensive drugs in new patients. J Hum Hypertens. 2002;16(6):439-444.

37. Park JH, Shin Y, Lee SY, Lee SI. Antihypertensive drug medication adherence and its affecting factors in South Korea. Int J Cardiol. 2008 128(3):392-398.

38. Friedman O, McAlister FA, Yun L, Campbell NR, Tu K. Canadian hypertension education program outcomes research taskforce. antihypertensive drug persistence and compliance among newly treated elderly hypertensives in ontario. Am J Med. 2010;123(2):173-181.

39. Hyre AD, Krousel-Wood MA, Muntner P, Kawasaki L, DeSalvo KB Prevalence and predictors of poor antihypertensive medication adherence in an urban health clinic setting. J Clin Hypertens. 2007;9(3):179-186.

40. Ben-Natan M, Noselozich I. Factors affecting older persons' adherence to prescription drugs in Israel. Nurs Health Sci. 2011;13(2):164-169.

41. Cárdenas-Valladolid J, Martín-Madrazo C, Salinero-Fort MA, et al Prevalence of adherence to treatment in homebound elderly people in primary health care: a descriptive, cross-sectional, multicentre study. Drugs Aging. 2010;27(8):641-651.

42. Piercefield EW, Howard ME, Robinson MH, Kirk CE, Ragan AP, Reese SD. Antihypertensive medication adherence and blood pressure control among central Alabama veterans. J Clin Hypertens. 2017; 19(5):543-549.

43. Armario P, Waeber B. Therapeutic strategies to improve control of hypertension. J Hypertens. 2013;31(Suppl 1):S9-S12.

44. Bangalore S, Shahane A, Parkar S, Messerli FH. Compliance and fixeddose combination therapy. Curr Hypertens Rep. 2007;9(3):184-189.

45. Ingersoll KS, Cohen J. The impact of medication regimen factors on adherence to chronic treatment: a review of literature. $J$ Behav Med. 2008;31(3):213-224.

46. Wang PS, Avorn J, Brookhart MA, et al. Effects of noncardiovascular comorbidities on antihypertensive use in elderly hypertensives. Hypertension. 2005;46(2):273-279.

\section{Dovepress}

CAS, Scopus and the Elsevier Bibliographic databases. The manuscript management system is completely online and includes a very quick and fair peer-review system, which is all easy to use. Visit http://www.dovepress. com/testimonials.php to read real quotes from published authors. 